\title{
BMJ Open Feasibility of implementing the advanced HIV disease care package as part of community-based HIV/TB activities: a mixed-methods study protocol
} Tinne Gils (D) , ${ }^{1}$ Lutgarde Lynen, ${ }^{1}$ Josephine Muhairwe, ${ }^{2,3}$ Kamele Mashaete, ${ }^{2}$
Thabo Ishmael Lejone, ${ }^{2}$ Philip Joseph, ${ }^{4}$ Thulani Ngubane, ${ }^{4}$ Alfred Kipyegon Keter, ${ }^{1}$
Klaus Reither, ${ }^{5,6}$ Alastair van Heerden ${ }^{4,7}$

\section{ABSTRACT}

Introduction Although the advanced HIV disease (AHD) care package reduces morbidity and mortality in people with AHD (defined in people living with HIV as WHO stage 3 or 4, CD 4 count $<200$ cells/ $/ \mathrm{L}$ or age $<5$ years), it is barely implemented in many countries. A novel pointof-care CD4 test rapidly identifies AHD. We evaluate the feasibility of implementing the AHD care package as part of community-based HIV/tuberculosis services.

Methods and analysis This two-phased study is guided by the Medical Research Council framework for evaluation of complex interventions. Stage 1 is a stakeholder consultation to define tools and indicators to assess feasibility of the AHD care package. Stage 2 is the implementation of the AHD care package during a facility-based tuberculosis diagnostic accuracy study in high-burden HIV/tuberculosis settings. Consenting adults with tuberculosis symptoms in two sites in Lesotho and South Africa are eligible for inclusion. HIV-positive participants are included in the feasibility study and are offered a CD4 test, a tuberculosis-lipoarabinomannan assay and those with $\mathrm{CD} 4$ count of $\leq 200 \mathrm{cell} / \mathrm{s} / \mathrm{L}$ a cryptococcal antigen lateral flow assay. Participants are referred for clinical management following national guidelines. The evaluation includes group discussions, participant observation (qualitative strand) and a semistructured questionnaire to assess acceptability among implementers. The quantitative strand also evaluates process compliance (process rating and process cascade) and early outcomes (vital and treatment status after twelve weeks). Thematic content analysis, descriptive statistics and data triangulation will be performed.

Ethics and dissemination The National Health Research and Ethics Committee, Lesotho, the Human Sciences Research Council Research Ethics Committee and Provincial Department of Health, South Africa and the Ethikkommission Nordwestund Zentralschweiz, Switzerland, approved the protocol. Dissemination will happen locally and internationally at scientific conferences and in peer-reviewed journals. Trial registration number NCT04666311. permitted under CC BY-NC. No commercial re-use. See rights and permissions. Published by BMJ.

For numbered affiliations see end of article.

Correspondence to

Tinne Gils; tinnegils@gmail.com

\section{INTRODUCTION}

Globally, AIDS-related deaths declined by $61 \%$ since 2004 due to the availability of HIV testing, antiretroviral treatment (ART) and early ART initiation. ${ }^{1}$ Unfortunately the HIV epidemic is
Strengths and limitations of this study

- This mixed-method study will be the first to evaluate the feasibility of implementation of the advanced HIV care package, including a novel point-of-care CD4 test.

- The study is embedded in a multicountry tuberculosis (TB) diagnostic accuracy study conducted in two sites in high-burden HIV/TB settings.

- The qualitative and quantitative tools for this study are prepared through extensive consultation with field staff, academic and programme experts.

- Convergent and holistic triangulation will be applied to data from semistructured questionnaires, group discussions, participant observation, process and early outcome evaluation.

- Due to COVID-19-related restrictions, the stakeholder consultation and part of the qualitative data collection are conducted online.

far from over, with 690000 (480 000-1 million) people living with HIV (PLHIV) dying from AIDS-related illness in 2020. ${ }^{1}$ Patients who present to, or re-enter care with advanced HIV disease (AHD) are at high risk of opportunistic infections and death, with a higher risk when immunity is lower. ${ }^{2}$ AHD is defined as present in PLHIV when having a WHO stage of 3 or 4 condition, a CD4 +Tcells (CD4) count below 200 cells $/ \mu \mathrm{L}$ or being a child $<5$ years old. ${ }^{3}$ AHD is common in sub-Saharan Africa, with studies reporting between $32 \%$ and $71 \%$ of patients initiating care with $\mathrm{AHD}$, and up to $60 \%$ patients presenting with AHD after disengagement. ${ }^{4-6}$ When initiating ART with AHD, patients have an estimated $17 \%$ risk of mortality within 1 year. $^{78}$ AHD requires prompt medical attention with up to $25 \%$ of patients with AHD with previous ART exposure dying within 48 hours of hospital admission. $^{5}$ 
Table 1 Overview of advanced HIV disease care package

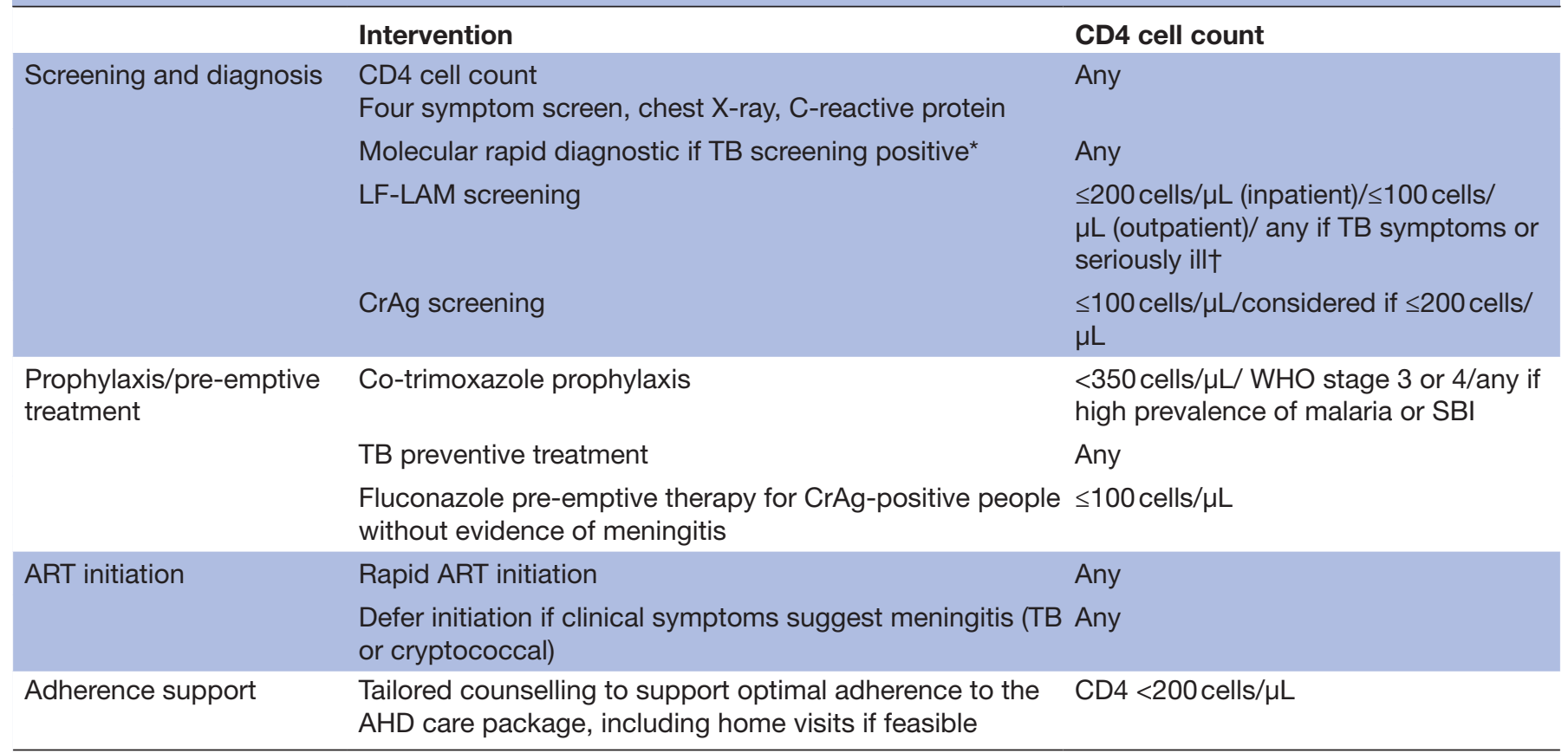

Adapted from $\mathrm{WHO}^{13}$

*WHO TB symptom screen includes presence of fever, weight loss or any cough. People living with HIV may present with signs or symptoms of extrapulmonary TB also including lymphadenopathy, meningitis or other atypical presentations warranting evaluation.

†Seriously ill is defined based on four danger signs: respiratory rate $>30 / \mathrm{min}$, temperature $>39^{\circ} \mathrm{C}$, heart rate $>120 / \mathrm{min}$ and unable to walk unaided.

AHD, advanced HIV disease; ART, antiretroviral therapy; CrAg, cryptococcal antigen; LF-LAM, lateral flow lipoarabinomannan assay; SBI, severe bacterial infections (including bloodstream, respiratory, central nervous system and gastrointestinal infections); TB, tuberculosis; WHO, World Health Organization.

Tuberculosis (TB), cryptococcal meningitis and severe bacterial infections are WHO stage 3 and 4 conditions that cause more than half of deaths among PLHIV. ${ }^{79} \mathrm{~TB}$ is the leading cause of AIDS-related deaths globally and its contribution is likely underestimated. ${ }^{10}$ In hospitals in resource-limited settings, TB remained undiagnosed at death in almost half $(45 \%)$ of HIV-positive TB cases. ${ }^{11}$ Cryptococcal meningitis accounts for $15 \%$ of AIDSrelated deaths globally, and $73 \%$ of prevalent cases are in sub-Saharan Africa. ${ }^{12}$

Since 2017, WHO recommends a package of interventions to manage AHD, including screening, treatment and/or prophylaxis for opportunistic infections, rapid ART initiation and intensified adherence support for those identified with AHD (table 1). ${ }^{313}$ This recommendation is based on evidence of the capacity of the package components to reduce morbidity and mortality among people with AHD. ${ }^{14-16}$ In absence of clinical indication of WHO stage 3 or 4 disease, a CD 4 count of 200 cells $/ \mu \mathrm{L}$ or less is the entry criterion to start the AHD care package in adults. When identified, AHD triggers screening for Mycobacterium tuberculosis lipoarabinomannan antigen (TB LAM) and cryptococcal antigen (CrAg) by use of point-of-care TB LAM and CrAg lateral flow assays, with patient management depending on results. ${ }^{3}$ In all HIVpositive patients with symptoms of TB, a sputum Xpert
MTB/RIF assay should be performed, along with a TB LAM test regardless of CD4 count. ${ }^{2} 1317$

The availability of point-of-care diagnostics, in addition to HIV tests, is thus essential for successful implementation of the AHD care package in primary care facilities. However, since the introduction of universal Test and Treat, CD4 measurement tests are no longer commonly available at primary care level. ${ }^{34}$ Moreover, TB LAM and CrAg lateral flow assays are not widely available in most low-income and middle-income settings. Consequently, the AHD care package is barely implemented. ${ }^{4}$ When present, the AHD care package is often only available to study populations and/or selected sites supported by international organisations. ${ }^{418}$

The novel Omega VISITECT CD4 Advanced Disease Lateral Flow Assay (Omega Diagnostics, Scotland, UK; VISITECT CD4 LFA), is the first disposable point-of-care test, which allows visual interpretation of a result of above or below $200 \mathrm{CD} 4$ cells $/ \mu \mathrm{L} .{ }^{19}$ The test had an acceptable diagnostic accuracy when performed by laboratory technicians on venous blood (sensitivity: $94.6 \%$, specificity: $81.7 \%$ ), and when performed by clinicians on finger-prick samples (sensitivity: $98.3 \%$, specificity $77.2 \%$ ) ${ }^{19}$ The test is also feasible and easy-to-use according to healthcare workers. ${ }^{19} 20$ 


\section{Box 1 Phases of framework for complex intervention}

Phase 0: Theory development: Identify the evidence base to select the appropriate intervention and identify and develop appropriate theory to develop the intervention.

Phase I: Formative stage: Identify the components of the intervention and the underlying mechanisms by which they will influence outcomes and link emerging perceptions to process indicators and outcomes indicators

Phase II: Assessing feasibility and piloting methods: Test procedures for compliance, acceptability and intervention delivery.

Phase III: Evaluation of the intervention: Study effectiveness of a fully defined intervention, ideally comparing with an appropriate alternative, using a protocol that is theoretically defensible, reproducible and adequately controlled in a study with appropriate statistical power.

Phase IV: Implementation: Determine whether others can reliably replicate your intervention and results in uncontrolled settings over the long term

Adapted from Campbell et al..$^{22}$

There are limited data on feasibility of AHD care package implementation, and on use of VISITECT CD4 LFA to identify AHD. ${ }^{2}{ }^{4}$ We integrate the AHD care package, including VISITECT CD4 LFA, into procedures of a prospective TB diagnostic accuracy study. We aim to evaluate the feasibility of implementing the AHD care package, including VISITECT CD4 LFA as part of community-based HIV/TB activities.

\section{METHODS AND ANALYSIS}

\section{The Medical Research Council Framework for complex} interventions

The intervention of introducing the AHD care package in community-based HIV/TB-programmes is considered complex, that is, contains several interacting components. The Medical Research Council framework is used for evaluation of this intervention (box 1)..$^{21} 22$

The content and benefits of the AHD care package are well defined and WHO recommended. Phase 0 is thus considered as accomplished. ${ }^{3}$ To assess the feasibility of implementing the AHD care package as part of community-based HIV/TB activities, we focused on phase I and phase II of the framework. This evaluation involves an iterative, cyclical course with multiple feedback loops, and integrates quantitative and qualitative methods. Phase III and IV have currently not been planned.

\section{Objectives}

The general objective is to evaluate the feasibility of implementing the AHD care package, including the novel VISITECT CD4 LFA, for implementation as part of community-based HIV/TB activities. The evaluation takes place in health facilities during a prospective diagnostic accuracy study and assesses the potential for implementation of the AHD care package in future community activities. The community-based HIV/TB activities could include interventions for prevention, diagnosis, treatment and/or care for HIV and/or TB taking place in the community, that is, not inside a health facility. The main research questions we address are:

- How is the AHD package implemented during a facility-based TB diagnostic accuracy study?

- Can the AHD care package be implemented as part of community-based HIV/TB activities?

- What is the best way to implement the AHD care package during community-based HIV/TB activities?

Among aspects of feasibility to be evaluated were acceptability among implementers, adherence to process flow and timing, and early outcomes. ${ }^{23}$

The sub-objectives per stage are:

\section{Stage I}

- Develop indicators and tools for evaluation of feasibility (acceptability, process, early outcomes) of implementation of the AHD care package including the novel VISITECT CD4 LFA.

- Evaluate and adjust draft materials for training and implementation support.

\section{Stage II}

- Evaluate the implementation process and early outcomes, identify barriers to implementation and possible solutions.

- Evaluate acceptability among implementers and identify necessary roles and responsibilities for implementation.

- Evaluate and adjust materials for training and implementation support.

Cross cutting objectives are to assess whether the feasibility of implementing the AHD care package differs according to the setting and its prevalence of detected AHD, and whether the feasibility is affected by the COVID-19 pandemic.

\section{Study design}

This is a two-site prospective study in two stages with a multi-strand (qualitative and quantitative), mixed-method multiphase design (figure 1). The two stages correspond to phases I and II of evaluation by the Medical Research Council. Stage I and II are sequential in time and stage I will provide exploratory data to develop tools used in stage II. Stage II includes a quantitative and qualitative strand of equal weight, with concurrent and sequential elements. Emerging data of one element influence the final data collection tools for other elements.

\section{Stage 1}

Stage 1 is an exploratory stakeholder consultation to prepare the implementation process, tools to be used, and the choice of evaluation and outcome indicators for stage 2. The process consists of information sharing, feedback loops and group discussions with iterative feedback.

Stage 2

Stage 2 is a feasibility study with a quantitative and a qualitative strand. The quantitative strand includes a process 


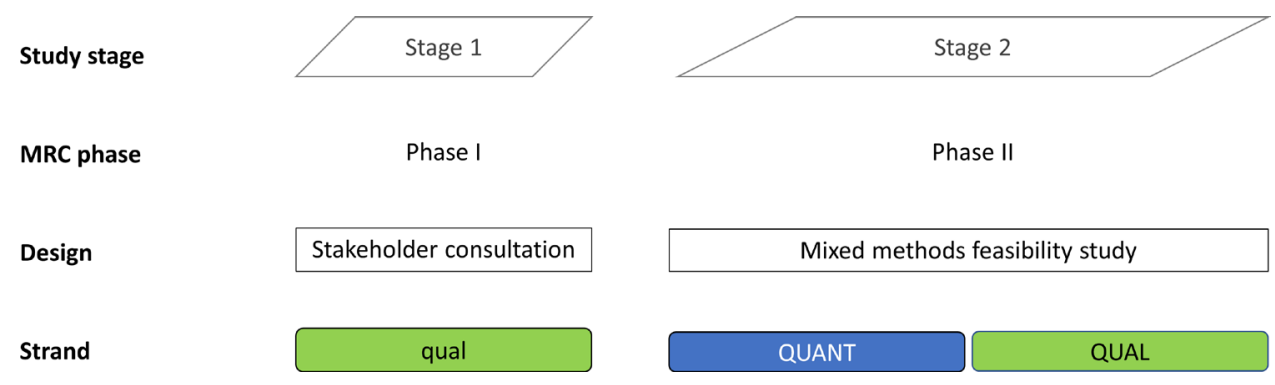

Figure 1 Diagram of study stages and methods. The strands include an exploratory qualitative strand with lower weight, followed by a quantitative and a qualitative parallel strand with equal weight. MRC, Medical Research Council; qual, qualitative; quant, quantitative.

cascade with data gathered during and in parallel with data collection of the diagnostic accuracy study, a semiquantitative rating of process steps, evaluation of early outcomes and assessment of feasibility indicators by means of a semistructured questionnaire. The qualitative strand includes participant observation by an expert evaluator and group discussions with implementers of the AHD care package. Data collection tools for qualitative and quantitative analysis can be adapted with emerging information from previous assessments.

\section{Study setting}

The stakeholder analysis is held online. The feasibility assessment takes place in two outpatient sites in South Africa and Lesotho and partly online.

\section{South Africa}

In South Africa in 2019, adult HIV prevalence was estimated at $19.0 \%(12.1 \%-24.7 \%)$, and incidence at 7.79 (4.58-10.80) per 1000 population. There were an estimated 84000 (43000-150 000) deaths of AIDS in 2019, with 36000 attributed to TB. Thirty-one per cent of patients had a CD4 count of $<200$ cells $/ \mu \mathrm{L}$ at diagnosis in the same year. ${ }^{24}$ The study is conducted in a mobile clinic outside Caluza Primary Healthcare facility in Pietermaritzburg, KwaZulu-Natal province, South Africa.

\section{Lesotho}

In Lesotho in 2019, the adult HIV prevalence was estimated at $21.8 \%(20.3 \%-21.9 \%), 8600(6300-9800)$ were newly infected and 4600 (3800-6100) died, with 3600 deaths attributed to TB. Twenty-four per cent of patients had a CD 4 count of $<200$ cells $/ \mu \mathrm{L}$ at diagnosis in the same year. ${ }^{24}$ The study is conducted at the premises of ButhaButhe District Hospital in Northern Lesotho.

\section{The AHD care package within the TB TRIAGE+ ACCURACY trial}

All study procedures and samples for TB TRIAGE +ACCURACY including those evaluated in the feasibility study are presented in online supplemental file 1. TB TRIAGE +ACCURACY plans to enrol 1400 participants. While in routine HIV/TB care, the entry point of the AHD care package would typically be a positive HIV status, in TB TRIAGE +ACCURACY, the entry point for patients is TB symptoms, after which an HIV test is done (in case of an unknown HIV status). Participants who are known HIV-positive cases or those who test HIV-positive in TB TRIAGE +ACCURACY are enrolled in the feasibility study. For those participants an Alere Determine TB LAM antigen test (Abbott, USA: Alere TB LAM) in urine is performed, and a serum CrAg lateral flow assay (IMMY, USA) in case of a CD4 count of 200 or less determined by VISITECT CD4 LFA. All patients enrolled in TB TRIAGE +ACCURACY receive a referral letter, including the results from AHD care package tests when applicable, for further follow-up and treatment by their healthcare provider according to national guidelines. ${ }^{25}{ }^{26}$ At 12 (10-14) weeks, patients receive a phone call to inquire about their vital status, TB and ART treatment status, and HIV-positive patients' treatment records are reviewed to determine the state of compliance with national guidelines for AHD care. All procedures restricted to HIVpositive participants are part of the feasibility study.

\section{The impact of COVID-19}

The surge of the COVID-19 pandemic since March 2020 led to protocol revisions in the preparatory phase. COVID-19 infection control precautions (masks, hand washing, optimisation of patient flow) were put in place at the study sites. Due to in-country and international travel restrictions, several trainings and the site initiation visits were conducted virtually. The stakeholder analysis, semistructured interviews and group discussions are held online. Assessment of the feasibility of implementing the AHD care package during the pandemic was included in the evaluation. Due to overlapping symptoms of TB and COVID-19, all patients in TB TRIAGE +ACCURACYalso receive a novel SARS-Cov-2 antigen rapid diagnostic test, a SARS-Cov-2 real-time PCR test and CAD4COVID, a digital chest X-ray analysis software, in combination with differential white cell count.

\section{Study population and recruitment}

Stage 1

The stakeholder consultation is conducted with international experts from the Swiss Tropical and Public Health Institute, the Institute of Tropical Medicine in Belgium, the Human Sciences Research Council in South Africa and SolidarMed in Lesotho, who are all coinvestigators to TB TRIAGE +ACCURACY. In addition, programme 
experts from the Human Sciences Research Council and SolidarMed are included.

\section{Stage 2}

The mixed-method study includes two study populations: patients and implementers.

\section{Patients}

The study population for evaluation of the process cascade and early outcomes are patients. Patients are screened for presence of any TB symptom at outpatient departments of the study sites and in the community. Those with $\mathrm{TB}$ symptoms are referred to the study facilities for TB TRIAGE +ACCURACY study procedures. Participants to TB TRIAGE +ACCURACYwho are HIV-positive receive the AHD care package. The following inclusion and exclusion criteria are applied for enrolment in TB TRIAGE +ACCURACY:

Inclusion criteria

- Willing and able to provide signed written consent or witnessed oral consent in the case of illiteracy, prior to undertaking any study-related procedure.

- Adults $(\geq 18$ years $)$.

- Any of the cardinal symptoms of TB (cough, weight loss, night sweats, fever) of any duration.

Exclusion criteria

- Pregnancy (based on oral information from participant).

- Any condition for which participation in the study, as judged by the investigator, could compromise the wellbeing of the subject, or prevent, limit or confound protocol specified assessments.

- Critically sick patients who need immediate medical care.

- Current anti-TB treatment.

Additional inclusion criteria for the feasibility study among those who are enrolled in TB TRIAGE +ACCURACY:

- Previously known HIV-positive or newly tested HIV-positive.

HIV testing is performed according to existing national guidelines. $^{25} 27$

\section{Implementers}

The study population for the process rating scale, structured questionnaires and participant observation are all health professionals or lay workers who implement the entire AHD package or parts of it, called the implementers. The expert evaluator, the person who oversees the AHD care package implementation process performs the process rating scale and participant observation and is additionally included in the group discussions. Written consent from participants is conditional to participation in group discussion and semistructured questionnaires.

\section{Outcomes}

The overall outcome is establishing the feasibility of implementing the AHD care package, including the VISITECT CD4 LFA, as part of community-based HIV/TB activities in Lesotho and South Africa. Specific outcomes per study stage were broadly defined in this protocol, and stage 1 outcomes are used to further elaborate stage 2 outcomes.

\section{Stage 1}

- Indicators and evaluation material on feasibility (including acceptability, process, and early outcomes) to evaluate pilot implementation.

- Training materials, standard operating procedures, job aids and other tools for implementation of the AHD care package.

\section{Stage 2}

Outcomes for the mixed-method study include qualitative and quantitative outcomes.

\section{Qualitative outcomes}

- Perceptions of implementers on feasibility of implementing the AHD care package during a facility-based TB diagnostic accuracy trial, as part of community HIV/TB activities, and during the COVID-19 pandemic.

- Perceptions of implementers on acceptability and feasibility of implementing the AHD care package for patients and for other healthcare workers.

- Barriers to and enablers for successful implementation.

- Requirements, roles and responsibilities of implementers.

\section{Quantitative outcomes}

- Feasibility and acceptability among implementers (proportions of answers to semistructured questionnaires).

- Process compliance (median scores on process rating scale, proportions of process steps fulfilled and timing of different steps).

- Early outcomes (vital status and proportion of patients for whom the AHD procedure led to the correct medical follow-up according to national guidelines after 12 weeks).

\section{Data collection}

Figure 2 presents an overview of data collection procedures in time and study populations.

\section{Stage 1}

The lead researcher performs a literature review and drafts indicators for feasibility, including acceptability and process, early outcomes and data collection tools. The lead researcher also drafts standard operating procedures, training materials and job aids for implementation. All drafts are shared electronically with the academic and programmatic experts accompanied by open and structured questions) aimed at identifying participants' preferences for certain indicators and the reasons why. Several drafts are shared, and adapted after feedback, through an iterative process. The process of semistructured feedback is complemented with online interviews with team members, to further explore anticipated barriers to 


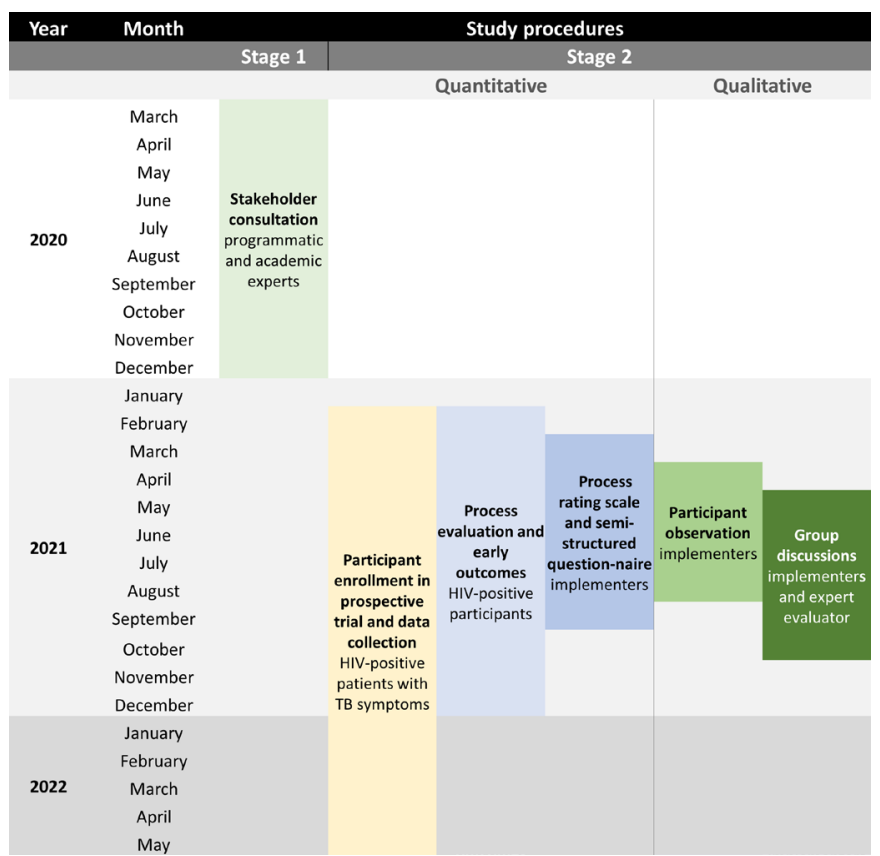

Figure 2 Overview of study procedures and population.

implementation, based on emerging themes from the questionnaires. An analytical memo is kept, detailing the dates, participants and outcomes of discussions and the decisions made.

\section{Stage 2}

\section{Process cascade}

Data on the different procedural steps and their outcomes are collected prospectively in MACRO trial software (MACRO V.4.8.1, Elsevier) during the cohort study. These data will be exported to Microsoft Excel to construct the process cascade.

\section{Early outcomes}

At 12 (10-14) weeks after enrolment, nurses within study teams conduct phone calls and extract data from patient records to ascertain their vital and treatment status. The data are entered in the MACRO database and will be exported to Microsoft Excel to construct a database for evaluation of early outcomes after AHD administration.

\section{Semistructured questionnaires}

A paper-based semistructured questionnaire, developed in phase I, is administered to all implementers of the AHD care package by the lead researcher. All participants receive an information sheet and sign informed consent before completing the questionnaire. The answers to the questionnaires are encoded in Microsoft Excel. The questionnaire is administered twice, after 3 and 6 months of AHD implementation. The content of the questionnaire for the 6-month evaluation can be adapted following new information that may arise during 3-month data collection.

\section{Process evaluation with rating scale}

An assigned expert evaluator from the study team will observe the implementers performing different steps of the AHD care package, monitor and record time spent on each step of the AHD procedure, and evaluate the completeness of the process, using a paper-based checklist with a Likert-type rating scale. The results will be encoded in Microsoft Excel.

\section{Participant observation}

To complement the quantitative evaluation with the rating scale, an expert evaluator gathers data resulting from participant observation of the people implementing the AHD care package, during the process. Depending on the situation, these observations may be participative (ie, from strict observation to asking questions during the process). The expert evaluator takes notes on paper during the observations and transcribes summaries of the observations in Microsoft Word documents.

\section{Group discussions}

The semistructured questionnaires and participant observations are complemented with group discussions, based on a topic guide developed from emerging themes from stage 1 and the previous steps in phase 2. All participants receive an information sheet and sign an informed consent before participating in the group discussion, including a separate consent for recording. The discussions are held in groups of up to eight people with similar profiles (study team). The lead researcher moderates the discussion online, assisted by an on-site moderator and translator who are united with the study team on site. Group discussions are conducted twice; once per site around 3 months after start of implementation, and once jointly with the implementers physically gathered at one of the sites. The main language is English, but participants can use isiZulu or Sesotho if they wish. The discussions are recorded on audiotape and a summary are transcribed on a Word document after the discussion by a trained and experienced transcriber/translator. A trained notetaker creates a memo describing expressions, gestures and other impressions that are not recordable.

\section{Patient and public involvement}

This feasibility study and the trial in which it is embedded was developed in consultation with service providers from Butha-Butha hospital and Caluza clinic, and field teams of SolidarMed and the Human Sciences Research Council. The field teams were direct stakeholders in the definition of study outcomes and study design. The study proposals were presented to the national TB programme in Lesotho and the provincial department of health in KwaZulu-Natal in South Africa. Patients were not directly involved in study design or conduct. However, study results will be disseminated locally to reach communities, including patients, civil society groups, and the wider 
public through presentations, local media and information on the websites of SolidarMed and the Human Sciences Research Council.

\section{Data analysis}

\section{Quantitative analysis}

Analyses will be performed using Stata/IC 16.0 (StataCorp). Participants' (patients and implementers) characteristics and outcomes from structured questionnaires will be summarised using means and SD or medians and IQRs for continuous variables, and frequencies and proportions for categorical data. Frequencies and proportions will also be used for description of the process cascade and early outcomes. Comparisons of means and medians will be done using t-test or nonparametric k-sample tests, respectively. Differences in proportions will be assessed using Pearson's $\chi^{2}$ tests or Fisher's exact test as appropriate.

\section{Qualitative analysis}

We will use an iterative approach, that is, collecting data and analysing through thematic content. Preliminary data will be used for further sampling, analysis and theory building. Formative data will be coded to inform further research questions and method application. Additional participant observation and group discussion may be conducted based on early results. Thematic content analysis will be used to categorise recurrent themes in transcripts of observations and group discussions. All transcripts and memos will be coded and analysed using QSR NVivo V.11 or higher.

\section{Triangulation}

We will apply convergent triangulation to validate the data obtained from different research methods and identify convergent results. We will also apply holistic triangulation to identify unique perspectives/angles that one or more of the individual methods can provide. Qualitative and quantitative data will initially be analysed separately as described above. Intermediate emerging information can be used to adapt tools and approach for future data collection (eg, if a missing process step is detected during the prospective cohort data collection, this challenge might be further explored during group discussions). At the end of the study, outcomes emerging from the different methodological strands will be compared for convergent and divergent information.

\section{ETHICS AND DISSEMINATION}

This study and the trial in which it is embedded, are being conducted in compliance with the approved protocols, the International Conference on Harmonisation Good Clinical Practice E6 and the current version of the Declaration of Helsinki. This protocol was approved by the National Health Research and Ethics Committee of Lesotho, the Human Sciences Research Council Research Ethics Committee, and the Provincial Department of Health of KwaZulu-Natal in South Africa, and the Ethikkommission Nordwest- und Zentralschweiz in Switzerland. Study implementers and expert evaluators provide written informed consent for participation in the structured questionnaires and group discussions. Patients enrolled in TB TRIAGE +ACCURACY provide informed consent, including for the AHD procedure and data collection. Both informed consent forms were translated in Sesotho and in isiZulu and back translated by a second person for quality control. SolidarMed and the Human Sciences Research Council will disseminate the information produced by this study locally through community advisory boards, provincial, district and national symposia, contacts to civil society groups, local press releases and information on the organisation website. The investigators will inform national health authorities about project results. Internationally, results will be disseminated through meetings with policy makers, publications in peer-reviewed journals, presentations at scientific conferences and through media.

\section{Author affiliations}

${ }^{1}$ Clinical Sciences, Institute of Tropical Medicine, Antwerpen, Belgium

${ }^{2}$ SolidarMed, Partnerships for Health, Maseru, Lesotho

${ }^{3}$ Institute of Global Health, University of Geneva, Geneva, Switzerland

${ }^{4}$ Centre for Community Based Research, Human Sciences Research Council,

Pietermaritzburg, South Africa

${ }^{5}$ Swiss Tropical and Public Health Institute, Basel, Switzerland

${ }^{6}$ Medical Outpatient Department, University of Basel, Basel, Switzerland

${ }^{7}$ Developmental Pathways for Health Research Unit, University of the Witwatersrand, Johannesburg, South Africa

Contributors TG and LL conceived the study idea. TG, JM, KM, TIL, PJ, TN, AKK and $\mathrm{AvH}$ designed the study. AvH, JM and KR supervised study implementation and data acquisition. KM, PJ and TN perform data acquisition and interpretation. TG, LL ad AvH drafted the first version of the manuscript. All authors revised the manuscript and agreed on the final version.

Funding This project is part of the European and Developing Countries Clinical Trials Partnership 2 (EDCTP2) programme supported by the European Union (grant number RIA2018D-2498).

Competing interests None declared.

Patient consent for publication Not applicable.

Provenance and peer review Not commissioned; externally peer reviewed.

Supplemental material This content has been supplied by the author(s). It has not been vetted by BMJ Publishing Group Limited (BMJ) and may not have been peer-reviewed. Any opinions or recommendations discussed are solely those of the author(s) and are not endorsed by BMJ. BMJ disclaims all liability and responsibility arising from any reliance placed on the content. Where the content includes any translated material, BMJ does not warrant the accuracy and reliability of the translations (including but not limited to local regulations, clinical guidelines, terminology, drug names and drug dosages), and is not responsible for any error and/or omissions arising from translation and adaptation or otherwise.

Open access This is an open access article distributed in accordance with the Creative Commons Attribution Non Commercial (CC BY-NC 4.0) license, which permits others to distribute, remix, adapt, build upon this work non-commercially, and license their derivative works on different terms, provided the original work is properly cited, appropriate credit is given, any changes made indicated, and the use is non-commercial. See: http://creativecommons.org/licenses/by-nc/4.0/.

ORCID iD

Tinne Gils http://orcid.org/0000-0002-5518-9600

\section{REFERENCES}

1 The Joint United Nations Programme on HIV/AIDS. Fact sheet 2021. Geneva: UNAIDS, 2021. Available: https://www.unaids.org/sites/ default/files/media_asset/UNAIDS_FactSheet_en.pdf [Accessed Sep 2021].

2 Ford N, Meintjes G, Calmy A, et al. Managing advanced HIV disease in a public health approach. Clin Infect Dis 2018;66:S106-10. 
3 World Health Organization. HIV treatment: guidelines for managing advanced HIV disease and rapid initiation of antiretroviral therapy: policy brief. Geneva: WHO, 2017. https://apps.who.int/iris/handle/ 10665/255885

4 Ndlovu Z, Burton R, Stewart R, et al. Framework for the implementation of advanced HIV disease diagnostics in sub-Saharan Africa: programmatic perspectives. Lancet HIV 2020;7:e514-20.

5 Ousley J, Niyibizi AA, Wanjala S, et al. High proportions of patients with advanced HIV are antiretroviral therapy experienced: hospitalization outcomes from 2 sub-Saharan African sites. Clin Infect Dis 2018;66:S126-31.

6 Calmy A, Ford N, Meintjes G. The persistent challenge of advanced HIV disease and AIDS in the era of antiretroviral therapy. Clin Infect Dis 2018;66:S103-5.

7 Walker AS, Prendergast AJ, Mugyenyi P, et al. Mortality in the year following antiretroviral therapy initiation in HIV-infected adults and children in Uganda and Zimbabwe. Clin Infect Dis 2012;55:1707-18.

8 Gupta A, Nadkarni G, Yang W-T, et al. Early mortality in adults initiating antiretroviral therapy (art) in low- and middle-income countries (LMIC): a systematic review and meta-analysis. PLoS One 2011;6:e28691.

9 Ford N, Shubber Z, Meintjes G, et al. Causes of hospital admission among people living with HIV worldwide: a systematic review and meta-analysis. Lancet HIV 2015;2:e438-44.

10 World Health Organization. Global tuberculosis report 2020. Geneva: WHO, 2020. https://www.who.int/publications/i/item/ 97892400131312021

11 Gupta RK, Lucas SB, Fielding KL, et al. Prevalence of tuberculosis in post-mortem studies of HIV-infected adults and children in resourcelimited settings: a systematic review and meta-analysis. AIDS 2015;29:1987-2002.

12 Rajasingham R, Smith RM, Park BJ, et al. Global burden of disease of HIV-associated cryptococcal meningitis: an updated analysis. Lancet Infect Dis 2017;17:873-81.

13 World Health Organization. Consolidated guidelines on HIV prevention, testing, service delivery and monitoring: recommendation for a public health approach. Geneva: WHO, 2021. https://www.who. int/publications/i/item/9789240031593

14 Mfinanga S, Chanda D, Kivuyo SL, et al. Cryptococcal meningitis screening and community-based early adherence support in people with advanced HIV infection starting antiretroviral therapy in Tanzania and Zambia: an open-label, randomised controlled trial. Lancet 2015;385:2173-82

15 Singhroy DN, MacLean E, Kohli M, et al. Adoption and uptake of the lateral flow urine Lam test in countries with high tuberculosis and HIV/AIDS burden: current landscape and barriers. Gates Open Res 2020;4:24.

16 Kimaro GD, Mfinanga S, Simms V, et al. The costs of providing antiretroviral therapy services to HIV-infected individuals presenting with advanced HIV disease at public health centres in Dar ES Salaam, Tanzania: findings from a randomised trial evaluating different health care strategies. PLoS One 2017;12:e0171917.

17 Huerga H, Rucker SCM, Bastard M, et al. Should Urine-LAM tests be used in TB symptomatic HIV-positive patients when no CD4 count is available? A prospective observational cohort study from Malawi. $J$ Acquir Immune Defic Syndr 2020;83:24-30.

18 Izco S, Murias-Closas A, Jordan AM, et al. Improved detection and management of advanced HIV disease through a community adult TB-contact tracing intervention with same-day provision of the WHO-recommended package of care including art initiation in a rural district of Mozambique. J Int AIDS Soc 2021;24:e25775.

19 Ndlovu Z, Massaquoi L, Bangwen NE, et al. Diagnostic performance and usability of the VISITECT CD4 semiquantitative test for advanced HIV disease screening. PLoS One 2020;15:e0230453.

20 Scorgie F, Mohamed Y, Anderson D, et al. Qualitative assessment of South African healthcare worker perspectives on an instrument-free rapid CD4 test. BMC Health Serv Res 2019;19:1-13.

21 Craig P, Dieppe P, Macintyre S, et al. Developing and evaluating complex interventions: the new medical Research Council guidance. Int J Nurs Stud 2013:50:587-92.

22 Campbell M, Fitzpatrick R, Haines A, et al. Framework for design and evaluation of complex interventions to improve health. BMJ 2000;321:694-6.

23 Bowen DJ, Kreuter M, Spring B, et al. How we design feasibility studies. Am J Prev Med 2009;36:452-7.

24 The Joint United Nations Programme on HIV/AIDS. Unaids data 2020. Geneva: UNAIDS, 2020. https://www.unaids.org/en/resources/ documents/2020/unaids-data

25 Republic of South Africa National Department of Health. National consolidated guidelines for the management of HIV in adults, pregnancy, adolescents, children and infants and prevention of mother-to-child transmission. Pretoria, 2020. Available: https:// www.knowledgehub.org.za/system/files/elibdownloads/202007/National\%20Consolidated\%20Guidelines\%2030062020\% 20signed\%20PRINT\%20v7.pdf [Accessed Sep 2021].

26 Ministry of Health, Government of Lesotho. Approach to the management of advanced HIV disease (AHD). Maseru, 2020. Available: https://differentiatedservicedelivery.org/Portals/0/adam/ Content/gDujY9rV90i3ggXjQ3Upow/File/Lesotho\%20Advanced\% 20HIV\%20Disease\%20Manual_June\%2025\%202020_vF.pdf [Accessed Sep 2021].

27 Ministry of Health, Government of Lesotho. National guidelines on the use of antiretroviral therapy for HIV prevention and treatment. Maseru, 2016. Available: https://www.childrenandaids.org/sites/ default/files/2017-04/Lesotho_ART-Guidelines_2016.pdf [Accessed Sep 2021]. 